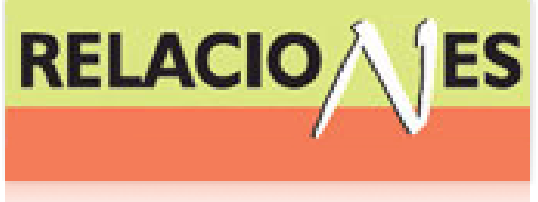

Relaciones. Estudios de historia y sociedad ISSN: 0185-3929

relacion@colmich.edu.mx

El Colegio de Michoacán, A.C

México

Pío Martínez, Juan

La ciencia de la nutrición y el control social en México en la primera mitad del siglo xx

Relaciones. Estudios de historia y sociedad, vol. XXXIV, núm. 133, 2013, pp. 225-255

El Colegio de Michoacán, A.C

Zamora, México

Disponible en: http://www.redalyc.org/articulo.oa?id=13725610008

Cómo citar el artículo

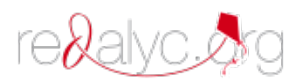

- Número completo

- Más información del artículo

Página de la revista en redalyc.org

Sistema de Información Científica

Red de Revistas Científicas de América Latina, el Caribe, España y Portugal Proyecto académico sin fines de lucro, desarrollado bajo la iniciativa de acceso abierto 


\title{
La ciencia de la nutrición y el control social en México en la primera mitad del siglo $\mathrm{XX}$
}

\author{
Juan Pío Martínez* \\ UNIVERSIDAD DE GUADALAJARA, CENTRO UNIVERSITARIO DE LOS LAGOS
}

El artículo inicia con un esbozo de cómo en Europa la ciencia de la nutrición devino ideología usada como instrumento de control social. En ese contexto se estudia el caso de México para analizar el vínculo de los médicos y el Estado con el propósito de transformar la alimentación popular. El objetivo es demostrar la vigencia de la ideología alimentaria introducida en México desde la época de la conquista y la consecuente paradoja de la ciencia de la nutrición, subordinada a la hegemonía cultural de las metrópolis occidentales.

(Nutrición, ideología, hegemonía, control social, alimentación popular)

\section{INTRODUCCIÓN}

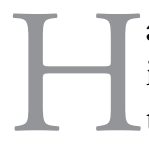

asta el momento ha sido poco estudiado el papel que las ideas sobre nutrición han desempeñado como instrumento de control social. ${ }^{1}$ Por lo general, tales ideas ni siquiera

*juanpiomtz@yahoo.com.mx

${ }^{1}$ Jeffrey Pilcher y Sandra Aguilar Rodríguez abordan esa temática. Para ambos autores el concepto de nutrición se convirtió en parte del discurso de las elites, que consideraban necesario modificar la dieta básica de la mayoría de los mexicanos, basada en alimentos autóctonos como el maíz, frijoles, chile y pulque, entre otros, para inducirlos a comer carne, leche y pan. Sin embargo, más que desarrollar ese aspecto, lo que a estos estudiosos les interesa es dilucidar el papel desempeñado por las mujeres en el desarrollo nacional. Véase, respectivamente, Jeffrey Pilcher, ; Vivan los tamales! La comida y la construcción de la identidad mexicana, trad. Victoria Schussheim, México, Ediciones de la Reina Roja, Conaculta, Ciesas, 2001; y Sandra Aguilar Rodríguez, "Alimentando a la nación: género y nutrición en México (1940-1960)”, en Revista de Estudios Sociales, núm. 29, Universidad de los Andes, abril 2008. Texto disponible en Internet: http://res. uniandes.edu.co/view.php/425/1.php, (s.p.) 
son motivo de reflexión, pues tiende a considerarse que si parten de una base científica entonces deben ser incuestionablemente ciertas. De esa manera se soslaya la necesidad de analizar antes que nada dicha base científica, en especial su carácter ideológico. Éste es el propósito del presente artículo, que parte de considerar el devenir de la ciencia de la nutrición en ideología, para luego estudiar brevemente su impacto en México. Se aborda la primera mitad del siglo xx porque es el tiempo en que la ciencia de la nutrición adquirió la madurez cuyas secuelas aún nos tocan. En el caso de México, la aplicación de las ideas sobre nutrición originadas a partir de la experiencia europea sirvió para forjar definitivamente un nacionalismo desprendido de sus tradiciones culinarias autóctonas. Al finalizar el periodo de estudio señalado, esas tradiciones quedaron como un símbolo de identidad nacional, pero en la práctica el progreso del país estuvo fincado en la dieta diseñada en Europa y en los Estados Unidos.

En ese sentido, el concepto de control social ${ }^{2}$ es entendido en este trabajo como un mecanismo de persuasión, pues a partir de las ideas nutricionales siguió vigente la pretensión de civilizar, según la perspectiva de la cultura occidental, a la mayor parte de la población mexicana. Al sugerirle a las masas como lo más científico y racional determinado patrón alimenticio, se buscaba controlar sus formas de alimentación y de paso justificar, como un proceso natural y necesario, el desarrollo económico y social propuesto por las clases gobernantes. Para argumentar al respecto se analiza primero la confrontación entre las perspectivas carnívora y vegetariana que destaca en el desarrollo de la ciencia de la nutrición. Más que la defensa subjetiva de una u otra postura, interesa demostrar que aun cuando

\footnotetext{
${ }^{2}$ Sobre lo polisémico del concepto de control social y la laxitud con que ha sido usado, véase: Morris Janowitz. “Teoría social y control social”, Traducción Juan Pegoraro. revisión de Máximo Sozzo, en American Journal of Sociology, vol 81, 1, Universidad de Chicago, 1975. Publicado en Delito y Sociedad. Revista de Ciencias Sociales, núm. 6/7, 1995. Texto disponible en Internet: http://www.catedras.fsoc.uba.ar/pegoraro/Materiales/Janowitz_Teoria_Social_Control_Social.PDF; también, Pedro Oliver Olmo. "El concepto de control social en la historia social: estructuración del orden y respuestas al desorden”, en Historia Social, núm. 51, 2005, 73-91. Texto disponible en Internet: http://www.uclm.es/profesorado/poliver/pdf/articulos/HistoriaSocial.pdf
} 
ambas llegaron a convertirse en formas ideológicas para defender sus planteamientos, la primera se impuso por una hegemonía cultural conformada en el mundo occidental desde sus orígenes. Esto hace imprescindible un breve esbozo al respecto.

Como es la carne de res la que destaca en la confrontación mencionada, cabe advertir que de ninguna manera se pretende sugerir que el control social estuviera orientado a propiciar entre las clases bajas el consumo de ese alimento. Desde esa perspectiva se calificaban como inferiores las prácticas alimenticias de estas clases, pero no se crearon las condiciones para que las mejoraran accediendo a ese consumo. Más bien, los nutriólogos y las clases gobernantes propusieron la leche de vaca como el alimento ideal para que los pobres contaran con proteína animal en su alimentación. Como el vegetarianismo tolera la inclusión de alimentos lácteos en el tipo de dieta que recomienda, parecería entonces que la discusión entre carnívoros y vegetarianos pasa a segundo término. Sin embargo, la cuestión es que no se trata solamente de los alimentos en sí. La carne y la leche requieren de la cría de ganado vacuno, con lo que la hegemonía de la perspectiva carnívora se mantuvo vigente al establecerse el control social con apoyo en la ciencia de la nutrición. Así, la carne de res continuó siendo el alimento de prestigio, aunque inalcanzable para los pobres, mientras se promovía que éstos adoptaran la leche como alimento sustituto. Ante los intereses económicos e ideológicos de los grupos en el poder, no se tuvo en cuenta la inadaptación fisiológica de la mayoría de los mexicanos para procesar la leche, ${ }^{3}$ y tampoco el agravante que representaba dedicar grandes extensiones de

${ }^{3}$ Como demostró Marvin Harris, los europeos fueron de los únicos habitantes del planeta que desarrollaron la enzima llamada lactasa para permitir la absorción de la lactosa que contiene la leche sin producir mayores estragos en el intestino delgado. Véase, Bueno para comer. Enigmas de alimentación y cultura, Colección Los Noventa, 75, México, Alianza, Conaculta, 1991, 174-201. Para el caso de los mexicanos, véase Sonia Corcuera de Mancera. Entre gula y templanza. Un aspecto de la historia mexicana. 2a. ed. México, Fondo de Cultura Económica, 1990, 84. Aún en la actualidad se afirma que en México "el $75 \%$ de la población mayor de seis años es intolerante a la lactosa". Mariano García Garibay y Agustín López Munguía Canales, "Enzimas inmovilizadas y su aplicación en la industria alimentaria", en Ciencia y desarrollo, México, Conacyt, número 58, septiembre-octubre de 1984 . No es casual que para continuar con el negocio se implementara el procesamiento de leche deslactosada. 
tierra a la ganadería vacuna y orientar una parte de los cultivos agrícolas a su sostenimiento, incrementando de esa manera la lucha por los cultivos básicos entre los animales y la población de bajos recursos que dependía de ellos. ${ }^{4}$ Claro que esto último es otra historia digna de un estudio aparte.

\section{LA CIENCIA DE LA NUTRICIÓN COMO IDEOLOGÍA}

En la mentalidad cristiana la confrontación entre carnívoros y vegetarianos se remonta al origen de la humanidad. En ese imaginario se muestra la existencia de una estratificación alimentaria, en la que el pan estaba reservado a la humanidad, mientras el animal sacrificado pertenecía a Dios. ${ }^{5}$ Siguiendo con el mito occidental, en un momento dado la humanidad hizo suyo el consumo de carne, la cual se mantuvo como un indicador diferencial: ascetas o guerreros, lascivia o abstinencia. ${ }^{6}$

Con todo y que el pensamiento cristiano sobre el origen del hombre se funda ante todo en mitos, el estudio de las sociedades europeas de la Edad Media corrobora ese carácter diferenciador del consumo de carne mencionado antes. En los inicios de esa etapa histórica las clases en el poder tuvieron en la comida la primera oportunidad para mostrar su superioridad. Desde entonces, la carne se convirtió "en el valor alimentario por excelencia". Un valor al que "los manuales de dietética posteriores al siglo $\mathrm{v}$ prestaron una atención mucho mayor", lo que indujo al pensamiento científico y a la opinión común a considerar a la carne como el "alimento natural" del hombre. Según esas ideas éste tenía el poder de nutrir el cuerpo, de endurecer sus músculos y darle al guerrero la fuerza y la legitimi-

${ }^{4}$ La situación fue recurrente en todo México, el resto del continente latinoamericano, incluso en otras partes del mundo, y se acentuó aún más en la segunda mitad del siglo xx. Véase al respecto, Ernest Feder, "La irracional competencia entre el hombre y el animal por los recursos agrícolas de los países subdesarrollados", en El trimestre económico, México, Fondo de Cultura Económica, número 185, enero-marzo de 1980, $49-84$.

${ }^{5}$ Amado Millán, "Acerca del status animal", en Ricardo Ávila, Dominique Fourier y Ma. Teresa Ruíz, comps., Estudios del Hombre 7, Guadalajara, Universidad de Guadalajara, Departamento de Estudios del Hombre, 1998, 135 y 137.

${ }^{6}$ Ibid., p. 136. 
dad para ejercer el poder. ${ }^{7}$ Esa mentalidad dio origen, desde los inicios de la llamada Baja Edad Media, a una "ideología alimentaria" que dividió a la sociedad en carnívoros y vegetarianos mediante el ejercicio del poder. ${ }^{8}$ Así, a partir del siglo xiv estaba bien establecido que la "calidad" de la comida tenía que ver con la "calidad" de la persona, por lo que era natural y necesario que los campesinos comieran alimentos de origen vegetal y la nobleza y burguesía alimentos de origen animal, como símbolo de poder y riqueza de estas últimas. Apoyo esencial de esos planteamientos fueron las teorías científicas de la época, los médicos y los literatos. ${ }^{9} \mathrm{Al}$ consolidarse en el Renacimiento esa ideología alimentaria y plantearse de una manera cada vez más consciente, ésta se arraigo en el imaginario de las clases dominantes. La burguesía del periodo pudo hacerla suya gracias al entrecruzamiento de esta clase y la aristocracia entre los siglos XIV al XVIII, de tal modo que mientras un sector se aburguesaba el otro se aristocratizaba. ${ }^{10}$

De esa manera, al tomar el poder en el siglo xix y dar inicio al sistema capitalista, la burguesía se apropió del proceso de civilización iniciado siglos antes, como si se tratara de algo nuevo y que respondía al impulso de esta clase. ${ }^{11} \mathrm{Y}$, al igual que la aristocracia, también hizo suyo el consumo de carne, como una forma de mostrar estatus y poder. Dado ese desarrollo histórico, fue común entre las clases altas europeas concebir a la alimentación carnívora como lo más propio de los pueblos civilizados. ${ }^{12}$ Bajo esa ideología, los llama-

${ }^{7}$ Massimo Montanari, El hambre y la abundancia. Historia y cultura de la alimentación en Europa, trad. Juan Vivanco, Barcelona, Crítica, Grijalbo, 1993, 22-25.

${ }^{8}$ Ibid., pp. 52-53.

${ }^{9}$ Ibid., pp. 88-91.

${ }^{10}$ José Luis Romero, Estudio de la mentalidad burguesa, México, Alianza, 1989, 39; Norbert Elias, El proceso de la civilización. Investigaciones sociogenéticas y psicogenéticas, 2a ed., trad. Ramón García Cotarelo, México, Fondo de Cultura Económica, 1994, 153.

${ }^{11}$ Elias, op. cit., pp. 140 y 148.

${ }^{12}$ A principios del siglo xIx, el gastrónomo Jean Anthelme Brillat-Savarin afirmaba que si aún existiera alguna nación vegetariana, "infaliblemente sería subyugada por ejércitos carnívoros". Jean Anthelme Brillat-Savarin, Fisiología del gusto, Barcelona, Optima, 2001, 147; Francisco Reuleaux, Los grandes inventos en todas las esferas de la actividad humana y sus principales aplicaciones cientificas, artísticas, industriales, comerciales y domésticas, tomo v, Primera traducción española, hecha de la octava edición alemana por Fe- 
dos de los vegetarianos a evitar ese tipo de alimentación resultaban infructuosos. Como dice Carson Ritchie, para la gente del siglo XIX era menos importante que la carne fuera un alimento rico en proteínas, "la razón que todos conocían es que la carne es un alimento de ricos". Alimentarse con un bistec o con una jugosa chuleta era "señal de prosperidad y el reconocimiento de un estatus, igual que el cuello almidonado, el abrigo bien cortado o el sombrero de copa". ${ }^{13}$ Como en siglos anteriores, esas eran ideas respaldadas en el desarrollo de la ciencia occidental. El reconocido químico alemán Justus von Liebig fue el primero en clasificar a mediados del siglo xIx la nutrición humana en tres categorías fundamentales: proteínas, carbohidratos y grasas. Las dos primeras servían para aumentar o reparar el organismo, las últimas eran usadas como combustible. ${ }^{14}$

Prácticamente a nadie parecía importarle las demostraciones científicas de finales del siglo XIX sobre lo perjudicial que podría resultar para la salud el consumo excesivo de carne, por lo que se sugería una mayor ingesta de frutas. ${ }^{15}$ Incluso el descubrimiento de las vitaminas contribuyó a poner en entredicho el imperio de lo que se consideraba como "ración alimenticia científica", basada en el número de calorías que proporcionan los alimentos de origen animal. Hasta llegó a pensarse en el fin de dicho imperio, como supuso el naturalista español y profesor de biología Orestes Cendrero $\mathrm{Cu}$ riel. ${ }^{16}$ Esto le dio a los nutriólogos afiliados al vegetarianismo argumentos más sólidos que los esgrimidos en tiempos anteriores, cuando las críticas a la voracidad carnívora de las clases dominantes partían de preceptos religiosos o morales. ${ }^{17}$ Con base en ellos se ata-

derico Gillman, Madrid, Gras y Compañía, editores, 1888, 372. Véase también, Carson I.A. Ritchie, Comida y civilización. De cómo los gustos alimenticios han modificado la Historia, trad. José Carlos Gómez Borrero, Madrid, Alianza Editorial, 1988, 258.

${ }^{13}$ Ritchie, op. cit., p. 260.

${ }^{14}$ George Rosen, $A$ history of public health, Nueva York, MD Publications, Inc., $1958,404$.

${ }^{15}$ En ese sentido fueron importantes las investigaciones del científico ruso Ilya Metchnikoff. Véase Ritchie, op. cit., p. 226.

${ }^{16}$ Orestes Cendrero Curiel, Elementos de higiene, 6a. ed. Santander, s.e., 1926, 148.

${ }^{17}$ Steven Chapin, "Historia y debate del vegetarianismo: sus raíces religiosas, médicas y filosóficas". Texto disponible en Internet: http://www.librosperuanos.com/traducciones/esquina32.html, (s.p); Montanari, op. cit., p. 148. 
caron dos de las ideas fundamentales defendidas por los carnívoros. La primera era la idea de que la carne cría carne. La segunda era la idea de que la carne ha sido universalmente considerada como el tipo de alimento nutritivo. Para los nutriólogos vegetarianos, "los verdaderos alimentos para producir la actividad muscular y cerebral de un modo regular y duradero, los que dan a la máquina humana la mayor producción con el mínimo de intoxicación, con el mínimo de fatiga y desgaste son los de origen vegetal" ${ }^{18}$ Precisamente a principios del siglo xx estaba comprobándose mediante investigaciones de laboratorio que una persona podía trabajar eficazmente y estar saludable manteniéndose con una dieta vegetariana. ${ }^{19}$

Además del aspecto científico vinculado con cuestiones fisiológicas y químicas, para algunos médicos defensores de la tendencia vegetariana el problema con el predominio de la alimentación carnívora tenía que ver con el desarrollo del capitalismo. Hacia 1942, el médico alemán Franz G. M. Wirz calificó al vegetarianismo nacido en Inglaterra a mediados del siglo XIX como "el primer intento histórico de lucha práctica contra la violencia ejercida por la economía a la alimentación natural". ${ }^{20} \mathrm{Y}$ es que Wirz consideraba un desatino el hecho de que desde aproximadamente 1840 empezara a dominar lo

${ }^{18}$ L. Pascault, La salud por la alimentación racional, Traducción del francés: José Roda Rodríguez, 3a ed. Barcelona, Librería de D. Ribó Pelayo, 1915, vi, 2 y 65-66.

${ }^{19}$ Véase, Casimiro Brugués. Química popular, 3a. ed., con un prólogo de José Casares, Barcelona, Gustavo Gili, editor, 1918, 343; Evans Newton, Percy T. Magan y George Thomason, El nuevo médico de la familia. Tratado sobre la prevención y curación de las enfermedades; no destinado a reemplazar al médico de la familia, sino a ayudar al lector a cooperar más inteligentemente con él, traducida al castellano por Marcelo I. Fayard y revisada por el Dr. Carlos E. Westphal, Mountain View, Pacific Press Publishing Association, 1925, 318; Henry C. Sherman, La ciencia de la nutrición, Versión española de Germán Somolinos, México, Fondo de Cultura Económica, 1945, 223-224; Cendrero Curiel, op. cit., p. 133.

${ }^{20}$ Franz G. M. Wirz. "Política sanitaria de la alimentación”, en Wilhelm Stepp, dir., Fisiología, patología, terapéutica y política sanitaria de la alimentación. Las vitaminas, Versión directa del alemán por el Dr. Julio G. Sánchez Lucas, Barcelona, Labor, 1942, 664. Aunque a Wirz se le identifica como un ideólogo nazi y un promotor del control social del pueblo germano, véase Corinna Treitel, "Nature and the nazi diet", en Food and foodways, 17:139-158, 2009, (texto recomendado en uno de los dictámenes al presente trabajo), en su artículo aquí citado no se encuentran manifestaciones que avalen esa caracterización. En última instancia, no es esa la faceta digna de rescatar, sino su contribución al señalar el control que el capitalismo adquirió sobre los mercados alimentarios. 
que llamó “dirección económica de la alimentación”, pues alimentar de un modo natural al hombre o a todo un pueblo, le parecía "difícilmente compatible con el objetivo de todo negocio lucrativo referido en este caso a la producción y al comercio de los alimentos, como si se tratara de la venta de medias, tabaco u objetos de metal". Cuanto más productivo sea el negocio, decía, tanto más restringida será la alimentación. No obstante, pese a la trascendencia que Wirz le concedía al vegetarianismo, no tuvo más remedio que reconocer que su importancia y efectos no podían compararse con los de su poderoso contrincante. ${ }^{21}$ Como este mismo autor sabía, uno de los problemas centrales en ese sentido era la hegemonía cultural de los carnívoros, pues los médicos seguían formándose, avanzado el siglo $\mathrm{xx}$, "con arreglo a la antigua doctrina energética y la doctrina dietética igualmente antigua y carente de fundamento", porque no tenía suficientemente en cuenta "el elevado nivel actual de las investigaciones hechas en los problemas de la alimentación, ni tampoco las necesidades de la alimentación pública”. ${ }^{22}$

Aparte de la formación de los médicos bajo la hegemonía de las ideas sobre nutrición de índole carnívora, habría que considerar también hasta qué punto el vegetarianismo podía tener una amplia aceptación entre la aristocracia y la alta burguesía, tan acostumbradas a moverse en un ambiente rico y opulento, reflejado en un consumo alimenticio basado en productos de origen animal. Éstos eran los grupos sociales que había que convencer de cambiar hábitos alimenticios pues eran, por otra parte, los que generaban la hegemonía cultural de la doctrina energética basada en proteína animal. Eso contribuía a catalogar a la alimentación vegetariana como una moda excéntrica entre la clase rica, a la vez que un símbolo de pobreza y fracaso entre la clase pobre. De ahí que los nutriólogos opuestos al vegetarianismo continuaran predicando la idea de que al comer debía buscarse hacer "carne con carne". ${ }^{23} \mathrm{Al}$ considerar que la dieta

${ }^{21}$ Ibid., pp. 663-664.

${ }^{22}$ Ibid., pp. 667-668.

${ }^{23}$ León Gerardin, El hombre. Elementos de fisiología, de higiene, de prehistoria y de etnografia, Versión castellana, París, México, Librería de CH. Bouret, 1903, 14; Emilio Bouant, Nuevo diccionario de química aplicada a las ciencias, a las artes, a la agricultura, a 
vegetariana impedía el desarrollo muscular requerido por la industria, pedían establecer un combate frontal contra sus defensores. ${ }^{24}$

Así pues, en la primera mitad del siglo xx, los estudios sobre nutrición siguieron orientados casi exclusivamente a establecer los valores calóricos y la cantidad de energía proporcionada por la comi$\mathrm{da}$, pese a evidenciar que la clase trabajadora se hallaba seriamente subalimentada en términos de los estándares nutricionales científicos, establecidos precisamente por la ciencia de la nutrición dominante. Hasta antes de la primera guerra mundial, los estudios al respecto tenían que ver con la pobreza y la eficiencia en el trabajo, pero a partir de esa conflagración bélica las investigaciones se reorientaron en torno a la investigación industrial y a la producción económica y de salud. Sin embargo, detrás de los exámenes sociales empíricos y las investigaciones psicológicas sobre la fatiga, resaltaba la metáfora del cuerpo como una máquina sujeta a las leyes de la termodinámica. Como escribió Bryan S. Turner: "La extensión del conocimiento (eugenésico, dietético, termodinámico) corresponde con el ejercicio del poder político sobre las clases trabajadoras". Se buscaba cuantificar para controlar mejor a los sectores sociales y a la vez controlar la producción de bienes materiales. ${ }^{25}$

la industria y a la farmacia, Traducido, anotado con datos referentes a Espańa y aumentado con gran número de artículos bajo la dirección de los señores Ramón de Manjarrés y Bofarull y Federico Trémols y Borrell; con la colaboración de Juan Antonio Vidal de Solano, Casimiro Brugués y Escuder y Ramón de Manjarrés y Pérez-Junguitu, Barcelona, Espasa y Compañía, editores, s.a., tomo I, p. 95; Julio Cantala, El insipido (Panorama del hombre civilizado), Nueva York, Press Service, Inc., 1941, 256; Pedro Escudero, La alimentación. Colaboraciones publicadas en La Prensa, Buenos Aires, Hachette, 1934, 41 y 75; Reuleaux, op. cit., pp. 370-371.

${ }^{24}$ Gerardin, op. cit., pp. 14-15; José Giral Pereira, Ración alimenticia desde los puntos de vista higiénico y social, Madrid, Librería Internacional, Adrián Romo, editor, 1914, 128-131; Josep Bernabeu-Mestre et al., "La alimentación como problema sanitario: nutrición y salud pública en la España de la primera mitad del siglo Xx", en VIII Congreso de la Asociación de Demografía Histórica, 30 de mayo, 1 y 2 de junio 2007, Maó (Menorca). Texto disponible en Internet: http://www.redmei.org/index.php?option=com docman\&task=cat_view\&gid=69\&dir=DESC\&order $=$ date $\& I t e m i d=45 \& l i m i t=5 \& l i m$ itstart $=10$, p. 28.

${ }^{25}$ Bryan S. Turner, "The discourse of diet", en Mike Featherstone, Mike Hepworth y Bryan S. Turner, eds., The body social process and cultural theory, Londres, Sage Publications, 1991, 167. 
Eso explicaría las características de la nueva cultura de la nutrición que surgió en el contexto internacional a partir de la década de 1920, según la cual la alimentación pasó a adquirir un efecto civilizador, principalmente sobre trabajadores y campesinos, las madres y sus hijos. ${ }^{26}$ En ese tiempo, la doctrina puramente energética de la alimentación basada en proteína animal se concebía como la "base fundamental de la dieta moderna" y el resultado de "un progreso científico enorme". Lo peculiar es que no perdió continuidad, a pesar de ser una doctrina considerada también como "desastrosa", por ser la causante de enfermedades por "carencia, raquitismo, trastornos intestinales con estreńimiento, etc.". ${ }^{27}$ La principal preocupación era definir la "dieta normal" para las poblaciones, preferentemente los trabajadores, más que resolver las carencias no solamente en términos de nutrientes, sino de alimentos o grupos de alimentos. ${ }^{28}$ En ese contexto, lo novedoso de la nutriología desde finales de los años veinte fue el establecimiento de una estrecha relación entre nutrición, agricultura, economía y salud pública. ${ }^{29}$ Esto llevó a la creación en 1945 de la Organización para la Agricultura y la Alimentación, FAO por sus siglas en inglés. Fue una especie de colofón que cerró la primera mitad del siglo xx con el proceso que constituyó a la ciencia de la nutrición en una ideología, la cual estaría al servicio del sistema productivo, orientado a satisfacer la necesidad de alimentos de origen animal.

${ }^{26}$ Bernabeu-Mestre, et al., op. cit., p. 19.

${ }^{27}$ Jaime Pi-Suñer, "El problema económico de la alimentación”, en Atenea. Revista mensual de ciencias, letras y artes, Santiago de Chile, Universidad de Concepción, núm. 79, septiembre de 1931, 406.

${ }^{28}$ José María Bengoa, "Nutrición en América Latina: algunos eslabones de su historia”, en Héctor Bourges R., José M. Bengoa y Alejandro M. O’Donnell, coords., Historias de la nutrición en América Latina, sLAN Sociedad Latinoamericana de Nutrición, Publicación SLAN número 1, Fundación Cavendes, INCMNSz Instituto Nacional de Ciencias Médicas de la Nutrición Salvador Zubirán, CESNI Centro de Estudios Sobre Nutrición Infantil. Texto disponible en Internet: http://www.slan.org.mx/docs/HistNut_SLAN. pdf, p. 16.

${ }_{29}$ Bernabeu-Mestre, et al., op. cit., p. 19, nota 26. 


\section{LA IDEOlOGÍA DE LA NUTRICIÓN EN MÉXICO}

En un país en que todavía hacia 1950 más de la mitad de la población vivía en comunidades rurales ${ }^{30}$ y se alimentaba de una manera predominantemente vegetariana, al igual que lo hacían las empobrecidas clases populares del medio urbano, poco impacto podía tener el movimiento vegetariano iniciado en Europa un siglo atrás. Menos aun cuando fue un movimiento que careció de respaldo de parte del cuerpo médico mexicano.

El momento de mayor esplendor de las ideas vegetarianas en México fue durante las primeras dos décadas del siglo xx. Y probablemente lo fue no tanto por el desarrollo de la ciencia de la nutrición, sino por la influencia del pensamiento asiático tan en boga desde el siglo anterior. ${ }^{31}$ Por otra parte, salvo contadas excepciones, fue un esplendor basado en la difusión del pensamiento de autores extranjeros y no precisamente en el desarrollo científico nacional. En general se refrendaban las ideas principales del vegetarianismo: por un lado, la idea del relativismo cultural para negar la universalidad de la alimentación carnívora; por otro, la idea de que la alimentación vegetariana "es infinitamente preferible, y produce mayor potencia, tanto en el cuerpo como en el alma”. ${ }^{32}$ Además de señalar que la carne era "la causa principal de las enfermedades y de la degeneración humana", se recurrió a la historia para demostrar "que los pueblos que no se alimentaban con carne, fueron antiguamente

${ }^{30}$ De la población total del país que ascendía en 1950 a 25,791,017 habitantes, $10,983,483$ era población urbana, representaba $42.6 \%$; y 14,807,534 era población rural, lo que a su vez representaba $57.4 \%$ del total. Véase Estadísticas históricas de México, $4^{\mathrm{a}}$ ed., tomo I, Aguascalientes, INEgI, 2000.. Texto disponible en Internet: http://www. inegi.org.mx/prod_serv/contenidos/espanol/bvinegi/productos/integracion/pais/historicas/EHM\%201.pdf, p. 31. El 61.8\% del total de la población padecía de desnutrición. Aguilar Rodríguez, op. cit., (s.p.)

${ }^{31}$ Eduardo Devés y Ricardo Melgar, "El pensamiento del Asia en América Latina. Hacia una cartografía”, en Revista de Hispanismo Filosófico, núm. 10, 2005. Texto disponible en Internet: http://descargas.cervantesvirtual.com/servlet/SirveObras/0159363 0980142818560035/022815.pdf?incr=1, pp. 7-8.

32 "La salud del cuerpo. El vegetarianismo", en El Maestro. Revista de Cultura Nacional, México, Universidad Nacional, núm. 2, 1921, 165 y 171-172; El Heraldo Naturista. Revista ilustrada de México, México, s.e., núm. 3, 1921-1922, 23. 
los más poderosos y civilizados, habiendo comenzado a decaer cuando empezaron a hacer uso de la carne en la alimentación". ${ }^{33}$ Así, con el apoyo de una peculiar noción de ciencia y otra de historia, se condenó el consumo de carne y se acusó a dicho consumo de generar la degradación moral de los individuos, al grado de volverlos viciosos, criminales y crueles. ${ }^{34}$

No obstante, en la primera mitad del siglo Xx, el vegetarianismo en México fue considerado una moda entre las clases altas, ${ }^{35}$ más que una alternativa sugerida por los nutriólogos para la población mexicana. La razón es que entre éstos predominaban los prejuicios sobre los alimentos autóctonos, los cuales respondían evidentemente a una mentalidad arraigada entre las clases altas desde la época del descubrimiento de América. Una mentalidad reforzada por la medicina en México, que seguía enseñando los principios de la doctrina energética constituidos en universales, al menos en el mundo occidental. Un caso que ilustra bien esa circunstancia, es el de José Quintín Olascoaga, uno de los principales ideólogos de la nutrición vinculados al Estado. Este médico se formó como dietista en Argentina con Pedro Escudero, para quien "la historia de los pueblos carnívoros y las experiencias que provocó el conocimiento de la alimentación de los mismos, demuestran la posibilidad de vivir en salud perfecta consumiendo únicamente productos animales". La carne le parecía a Escudero un alimento fundamental para el hombre, por lo que podía consumirla generosamente, sin "ningún inconveniente". ${ }^{36}$ En 1939, cinco años después de la publicación de

33 "La alimentación natural”, en El Maestro, op. cit., número vi, 1922, 601.

${ }^{34}$ Ibid., p. 602; véase también "La salud del cuerpo. El vegetarianismo", op. cit., p. 169; El Heraldo Naturista. Revista ilustrada de México, México, s.e., núm. 2, 1921-1922, 39-40; Ibid., núm. 7, 1923-1924, 33.

${ }^{35}$ Jaime Pi-Suñer Bayo, Las bases fisiológicas de la alimentación, México, Fondo de Cultura Económica, La Casa de España en México, 1940, 163; El Hogar, México, Compañía Editora Mexicana, núm. 1066, 13 de marzo de 1941, 32.

${ }^{36}$ Escudero, op. cit., pp. 54 y 61. Pedro Escudero fue el primero en crear en 1938 un Instituto Nacional de la Nutrición, donde además de mostrar su liderazgo intelectual y científico se formaron la gran mayoría de los médicos y dietistas de América Latina de aquellos años. Isaias Schor y Saul Senderey, "Nutrición siglo xx. Bosquejo histórico de la nutrición en la República argentina”, en Bourges, Bengoa y O’Donnell, op. cit., pp. 71- 
esas ideas, Olascoaga publicó un artículo en el que citó el libro de Escudero mencionado antes, bajo el argumento de que era el "tipo de encuesta individual" y "uno de los apoyos más serios desde el punto de vista experimental y no simplemente teórico, que sirven para demostrar que la carne no es nociva al hombre sano, sino por el contrario es indispensable". Es “totalmente inocua”, incluso si sólo se consume carne por un tiempo largo, "sin que ella traiga la menor molestia en los individuos sanos". ${ }^{37}$

En coincidencia con Escudero, también los médicos mexicanos invocaron a la historia para criticar la alimentación vegetariana y afirmar que lo inherente a la civilización humana era la alimentación carnívora. ${ }^{38}$ Según Alfredo Ramos Espinosa, otro de los más importantes ideólogos de la nutrición al servicio del Estado, la realidad histórica "nos muestra cómo los pueblos mejor alimentados, los que disponen de una alimentación variada, equilibrada y completa son los eternos dominadores y conquistadores de los que viven tristemente comiendo maíz y algunas hierbas". Para este médico, el problema de los indígenas nada tenía que ver con cuestiones de "inferioridad humana", sino con su inadecuada alimentación, secularmente sostenida hasta mediados del siglo xx. ${ }^{39}$ Desde su punto de vista, las poblaciones indígenas sufrieron una tragedia, al tener que vivir "sin ganadería, sin carnes, sin leches", porque esto hizo que fueran poblaciones "que a través de la historia se encogen, se achaparran y desaparecen". ${ }^{40}$

Bajo esos principios la hegemonía de los ideólogos de la nutrición en México se manifestó con mayor fuerza entre las décadas de

72. Véase también, José María Bengoa Lecanda, "Nutrición en América Latina: algunos eslabones de su historia”, en Bourges, Bengoa y O’Donnell, op. cit., pp. 16 y 31.

${ }^{37}$ José Quintín Olascoaga, "Bases para los estudios de bromatología y nutrición en la República Mexicana”, en Medicina. Revista mexicana, México, Escuela Nacional de Medicina, núm. 348, septiembre 25 de 1939, 387-388.

${ }^{38}$ Véase Francisco de P. Miranda, La alimentación en México, México, Instituto Nacional de Nutriología, 1947, 7-8; Pi-Suñer Bayo, op. cit., pp. 166-167.

${ }^{39}$ Alfredo Ramos Espinosa, La alimentación en México, México, s.e., 1939, 33 y 129.

${ }^{40}$ Alfredo Ramos Espinosa, "Mi contribución al estudio de la alimentación mexicana”, en Medicina. Revista Mexicana, México, Escuela Nacional de Medicina, tomo xxviII, núm. 560, julio 25 de 1948, 325-326. 
1930 y 1940, como resultado de la dirección política y económica que tomaba el país, cada vez más orientada a los procesos de industrialización y de urbanización. Por eso los médicos veían en la mayor parte de los mexicanos carencias alimenticias y nutritivas, expresadas en el bajo consumo de calorías provenientes de las proteínas animales y en las deficiencias de peso y talla, comparadas con los parámetros europeos y estadounidenses. Debido a ello, la dieta fue vista como una de las principales causas de la pobreza de las clases populares y del atraso económico del país. Se pensó que al mejorar esa carencia se crearían trabajadores sanos, disciplinados y productivos, lo que garantizaría el progreso de la nación. ${ }^{41}$ En función de ese objetivo, los médicos y el Estado se dieron a la tarea de combatir lo que consideraban problemas de desnutrición en México. Para ello se crearon una serie de instituciones a partir de 1936 encargadas de investigar ese problema y de diseñar a la vez estrategias para resolverlo. Lo destacable de ese proceso fue su desarrollo paradójico. Por un lado, la investigación científica destruyó mitos en torno a las descalificaciones sobre las cualidades nutritivas de los alimentos autóctonos, secularmente utilizados por los indígenas; por el otro, se tendió a ignorar los hallazgos positivos de dichas cualidades debido a prejuicios profundamente arraigados en el imaginario dominante.

\section{LOS NUTRIÓLOGOS Y LA ALIMENTACIÓN POPULAR}

El término "alimentación o nutrición popular" fue usado desde 1935 para referirse al proyecto del Estado orientado a tratar el tema de la alimentación como un problema de salud pública. ${ }^{42}$ Un proyecto que no sólo tomaría en cuenta el aspecto del abasto alimentario, sino que incluiría la idea de nutrir al individuo siguiendo los

${ }^{41}$ Véase al respecto Aguilar Rodríguez, op. cit., (s.p)

${ }^{42}$ Jesús Díaz Barriga, "La ración alimenticia de las masas campesinas y obreras", 1935. citado (s.p.i.) por Sara Aguilera Ríos y Juan José Saldaña, "Estado, comunidad médica e institucionalización de los estudios sobre nutrición en México (1937-1957)", en Juan José Saldaña, coord., La Casa de Salomón en México. Estudios sobre la institucionalización de la docencia y la investigación científicas, México, Facultad de Filosofía y Letras, UNAM, 2005, 375. 
principios que dictaba la ciencia, para lo cual se crearon una serie de instituciones conducentes a ello. El término de alimentación popular aludía al tipo de alimentación de las clases pobres, compuestas en su mayor parte por mestizos y en menor medida por indígenas, pero también puede considerarse un eufemismo para referirse al modelo de alimentación indígena. De hecho, las publicaciones de los médicos de la época lo confirman.

El interés gubernamental por la alimentación y nutrición de las clases populares, como parte de un programa enfocado al control social de las mismas, inició en 1935 con la creación de la Oficina de Higiene de la Alimentación, que al año siguiente pasó a ser la Comisión Nacional de la Alimentación, a cargo de Olascoaga. Ésta fue creada con el propósito, "intentado por primera vez, de estudiar la alimentación mexicana en todos sus aspectos" ${ }^{43}$ La susodicha comisión duró hasta 1942, cuando el propio Olascoaga, bajo el gobierno del general Manuel Ávila Camacho, planeó la organización del Instituto Nacional de Nutriología (INN), el cual empezó a desarrollar sus funciones en forma sistemática el 1 de enero de 1943, bajo la dirección del médico Francisco de P. Miranda. ${ }^{44}$ La relevancia del INN para este artículo estriba en su carácter social y su estrecha vinculación a los intereses del Estado para el control social de las clases populares. El mismo Olascoaga explicó que, entre otras funciones, el Instituto debía servir de guía al pueblo mexicano, "para orientarlo hacia la alimentación racional, económica e higiénica, de acuerdo con los hábitos y gustos que le son peculiares”. También serviría a los intereses del Estado, pues los resultados de su trabajo de investigación estarían al servicio de éste, "para orientar la política nacional en materia de alimentación humana de asistencia social por medio de la alimentación y de la producción de alimentos, el intercambio, etc.". 45

${ }^{43}$ Historia de la salubridady de la asistencia en México, tomo III, México, Secretaría de Salubridad y Asistencia, 1960, 733; Aguilera Ríos y Saldaña, op. cit., p. 376.

${ }^{44}$ José Quintín Olascoaga, "Datos para la historia de la nutriología en México”, en Medicina. Revista Mexicana, México, Escuela Nacional de Medicina, núm. 573, febrero 10 de 1949, 17.

${ }^{45}$ Olascoaga, loc. cit. 
Puesto que la llamada alimentación popular consistía principalmente de maíz, frijoles, chile y pulque, suplementada con arroz cada vez en mayor medida, los investigadores del INN se concentraron en estudiar las cualidades nutritivas de esos alimentos. Entre éstos, el maíz y el pulque fueron los artículos alimenticios que mayores discusiones ideológicas suscitaron. Sin embargo, por cuestión de espacio sólo se trata aquí la discusión a que dio lugar el estudio sobre el maíz y la forma en que fue desacreditado por los médicos mexicanos. ${ }^{46}$ De entrada, los análisis bromatológicos pusieron al descubierto que la importancia del maíz radicaba en su combinación con el frijol y el chile, formando una dieta que proporcionaba las cantidades adecuadas de todos los nutrientes esenciales. Esto hizo escribir a Francisco de P. Miranda, que la superioridad del trigo sobre el maíz no era tan grande como se había supuesto, y a William D. Robinson, investigador de la Fundación Rockefeller, que esa era una dieta "mucho más satisfactoria de lo que se había pensado hasta ahora" ${ }^{47}$ En 1949 , empezaron a revelarse los componentes químicos del maíz. De éstos cabe destacar la mención del triptófano, el ácido fólico o factor antianémico, la niacina y la riboflavina. ${ }^{48}$ Estos últimos porque por entonces todavía se creía que la niacina y la riboflavina eran sustancias que sólo se encontraban en la carne, la primera, y en la leche y los huevos, la segunda.$^{49}$ De los otros se hará referencia más adelante, al tratar el tema de la pelagra.

\footnotetext{
${ }^{46}$ El chile también fue motivo de polémica, pero los embates contra su producción y su consumo no tuvieron la misma intensidad que el maíz y el pulque. En cuanto a los frijoles se refiere, básicamente no hubo problema en cuanto a ser un producto contrastante con la cultura alimentaria occidental. Ya los espańoles estaban familiarizados con esta legumbre desde antes del descubrimiento de América, "y no podemos hablar propiamente de la introducción de una nueva variedad botánica”. Pedro Plasencia, A la mesa con don Quijote y Sancho, España, Punto de lectura, 2005, 17. Lo que no los despojó a la larga del estigma de ser un alimento de pobres.

${ }^{47}$ Pilcher, op. cit., p. 148.

48 Olascoaga mencionó la lisina "y los otro ocho aminoácidos esenciales". "Datos para la historia...”, op. cit., p. 19. El ingeniero químico René O. Cravioto encontró proteínas, cenizas, calcio, fósforo, fierro, caroteno, extracto no nitrogenado, tiamina, niacina y riboflavina. René O. Cravioto, "Contribución al conocimiento del valor nutritivo de los alimentos mexicanos: el maíz", en Medicina. Revista Mexicana, México, Escuela Nacional de Medicina, núm. 584, julio 25 de 1949, 293 y 295-296.

${ }^{49}$ Archivo Secretaría de Salubridad y Asistencia, DF, fondo SSA, sección SubSyA, caja 17, exp. 11.
} 
Pese a tales descubrimientos los médicos de la época consideraban que a la tortilla, para que fuera un buen alimento, debía agregársele alimentos de origen animal. ${ }^{50} \mathrm{Y}$ es que en el discurso oficial se mantuvo como ideal "la adopción de alimentos tales como la carne de res y la leche, en vez de impulsar el consumo de frutas y vegetales silvestres y regionales, ya que éstos eran asociados con la cultura indígena y campesina". Esa actitud muestra, sobre todo, "que el valor de los alimentos no estuvo determinado tan sólo por la cantidad de nutrientes que contenían, sino principalmente por las ideas y prácticas identificadas con los grupos que las consumían". ${ }^{51}$ Eso explica la insistencia de incluir la leche en la dieta popular. Una propuesta que no sólo iba dirigida a los grupos mestizos urbanos y rurales, sino incluso a comunidades indígenas. Se pensaba que la adición de un vaso de leche al día subsanaría las deficiencias que la perspectiva prejuiciada de los investigadores encontraba en una dieta a base de tortilla, frijol, chile, pulque y algunos vegetales verdes. ${ }^{52}$ El hecho de que los médicos e ingenieros químicos mexicanos pasaran por alto las consecuencias gastrointestinales que conllevaba el consumo de tortillas y leche, ${ }^{53}$ sólo demuestra la existencia en ellos

${ }^{50}$ Norberto Treviño. "Alimentación en los trabajadores", en CAMEP. La Prensa Médica Mexicana, México, Centro de Asistencia Médica para Enfermos Pobres, número 12, 1 de noviembre de 1936, 163-164; Epaminondas Quintana, "El ingente problema del maíz en su aspecto agrícola y nutritivo", en América indigena. s.p.i., número 2, abril 1944, 131; Alfredo Ramos Espinosa, "Industrialización del maíz", en Medicina. Revista Mexicana, México, Escuela Nacional de Medicina, núm. 586, agosto 25 de 1949, 125-126; Ramos Espinosa, "Valor energético medio de la alimentación en México", en Medicina. Revista Mexicana, México, Escuela Nacional de Medicina, núm. 292, mayo 25 de 1937, 244; Miranda, op. cit., p. 21.

${ }^{51}$ Aguilar Rodríguez, "Alimentando a la nación...”, op. cit., (s.p).

${ }^{52}$ Rene O. Cravioto, P. G. Massieu, Jesús Guzman G., "El problema de las proteínas en la dieta mexicana”, en Boletín de la Oficina Sanitaria Panamericana, febrero 1955. Texto disponible en Internet: http://hist.library.paho.org/Spanish/BOL/v38n2p148. pdf, p. 152. El intento de sustituir el pulque por la leche en el Valle del Mezquital fracasó, porque el nuevo alimento les provocó trastornos gastrointestinales a los habitantes de ese lugar. Véase, Gonzalo Aguirre Beltrán, Obra antropológica v. Programas de salud en la situación intercultural, México, Universidad Veracruzana, Instituto Nacional Indigenista, Gobierno del Estado de Veracruz, Fondo de Cultura Económica, 1994 (Primera edición, III, 1955), p. 91.

${ }^{53}$ Alfredo Ramos Espinosa sabía que la forma de preparar el nixtamal a base de maíz y cal, era la causa de que los habitantes de las poblaciones rurales tuvieran diarrea cuando 
de una tendencia ideologizada, orientada a imponer patrones de consumo desde un punto de vista unidimensional y sin atender, por lo mismo, a la alteridad.

Aun cuando seguía generando sorpresa la capacidad de supervivencia de los pueblos indígenas de México alimentados básicamente con maíz, se siguió culpando al consumo de este cereal del atraso económico y cultural de México. ${ }^{54} \mathrm{El}$ problema, sin embargo, no estaba precisamente en el consumo de ese cereal, sino en la falta de acceso a la tierra, a la educación y a las fuentes económicas. ${ }^{55} \mathrm{De}$ cualquier manera, los ideólogos de la nutrición empezaron a investigar en la década de 1940 las posibilidades de sustituir el maíz por la soya, como fuente de proteínas para aquellos sectores que no podían acceder a la proteína animal, por falta de recursos económicos. En realidad, el proyecto de sustituir el maíz por algún otro alimento no era nuevo; se dejó de pensar en sustituirlo por el trigo cuando las investigaciones bromatológicas empezaron a demostrar que existía casi una equivalencia nutritiva entre ambos cereales. ${ }^{56}$ Entonces se pensó en la soya, pero tampoco era nueva la propuesta de sustituir el maíz por esa leguminosa. ${ }^{57}$ En todo caso, lo novedoso fue la injeren-

no se atendía al aserto de que "la tortilla no circula con leche". Alfredo Ramos Espinosa, "El folklore y la alimentación", en Anuario de la Sociedad Folklórica de México. 1941, vol. II, México, Círculo Panamericano de Folklore, 1943, 196; también, Ramos Espinosa, "El maíz, el frijol y el chile", en CAMEP. La Prensa Médica Mexicana. México, Centro de Asistencia Médica para Enfermos Pobres, núm. 2, sábado 15 de febrero de 1941, 17.

${ }^{54}$ Quintana, op. cit., p. 129; Ramón Fernández y Fernández, "Las posibilidades agrícolas de México", en Universidad mensual de cultura popular, México, Universidad Nacional, núm. 10, noviembre de 1936, 35; Revista de investigación clínica. Órgano del Hospital de Enfermedades de la Nutrición, México, Hospital de Enfermedades de la Nutrición, núm. 1, octubre de 1948, 128. Rubén García, "Hambre y maíz. Síntesis del artículo del Gral...., publicado en El Universal”, en Guión, México, s.e., núm. 5, 1 de marzo de 1939,17 y 19.

55 Sandra Aguilar Rodríguez, "Cooking modernity: nutrition policies, class, and gender in 1940s and 1950s Mexico city", en The Americas, 64:2 octubre 2007, 182.

${ }^{56}$ Pilcher, op. cit., pp. 143-144 y 148. Desde sus primeros escritos, Alfredo Ramos Espinosa abogó porque el maíz fuera desplazado por otros alimentos: "cereales distintos, legumbres, frutas, carnes, leche, mantequilla, huevos, todo de buena calidad". Alfredo Ramos Espinosa, "Notas breves sobre nuestras posibilidades de alimentación", en Medicina. Revista Mexicana, México, Escuela Nacional de Medicina, núm. 265, abril 10 de 1936, 147.

${ }^{57}$ Desde 1929, Manuel Gamio vivió empeñado en realizar ese cambio. Para él, la 
cia del Estado en colaboración con el INN para promover, a partir de 1950, programas con el objetivo de fomentar la aceptación de la harina de nixtamal enriquecida con soya, así como la fabricación de atole de maíz y soya u horchata o leche de la misma leguminosa. ${ }^{58}$

Como apoyo a los intentos para sustituir al maíz, se le acusaba también de causar la pelagra, una enfermedad descubierta en Europa desde 1730 por el doctor Gaspar Casal y cuyos síntomas fueron sintetizados como las tres "D": dermatitis, diarrea y demencia. ${ }^{59}$ Entre los principales promotores de esa acusación estaban Alfredo Ramos Espinosa ${ }^{60}$ y Francisco de P. Miranda. Este último, con todo y haber reconocido que el trigo no era realmente superior al maíz, insistía en que éste era deficiente en niacina, que es "la vitamina preventiva de la pelagra”; también decía que era deficiente en triptófano, lo que a su parecer se añadía "para el efecto de la producción de pelagra" ${ }^{61}$ Lo interesante es la existencia de versiones contrarias al respecto. En 1863 el médico Ismael Salas demostró que aun pese a que las clases pobres vivían casi exclusivamente de maíz, nunca se había presentado ese mal. ${ }^{62}$ Entre 1942 y 1945 algunos investigadores de la Fundación Rockefeller y de la Fundación Kellogg encontraron en México sólo síntomas subclínicos entre los campesinos más pobres, "porque la técnica de remojar el maíz en agua con cal liberaba gran parte de la niacina, cuya escasez en el maíz se suponía

soya tiene "un poder alimenticio muy superior al de la carne, huevos, leche y pan". Véase su obra, Antología, Estudio preliminar, selección y notas por Juan Comas, 2a ed., México, unAM, 1985, 54 y 59 y 66-67. También, Pilcher, op. cit., pp. 121 y 141.

${ }^{58}$ Archivo Secretaría de Salubridad y Asistencia, fondo ssa, sección SubSyA, caja 17, exp. 11 .

${ }^{59}$ En Oviedo la llamaban "mal de la rosa”, y los campesinos lombardos, pelagra, nombre que difundió el médico Francesco Frapolli, en un tratado publicado en 1771. Arturo Warman, La historia de un bastardo: maiz y capitalismo, México, Fondo de Cultura Económica, UNAM, 1988, 149-150.

${ }^{60}$ Alfredo Ramos Espinosa, "La habitación y el vestido en el campo.- La alimentación popular y los procesos mentales", en Medicina. Revista Mexicana, México, Escuela Nacional de Medicina, núm. 252, septiembre 25 de 1935, 463; del mismo autor, "En torno al estudio geográfico de la alimentación en México", en Medicina. Revista Mexicana, México, Escuela Nacional de Medicina, núm. 440, julio 25 de 1943, 288; y, "El maíz, el frijol..." op. cit., p. 19.

${ }^{61}$ Miranda, op. cit., pp. 21 y 26.

${ }^{62}$ Warman, op. cit., p. 153. 
era la causa de dicho mal". ${ }^{63}$ Incluso el médico José Calvo de la Torre, a pesar de que también a él le parecía el maíz defectuoso en lisina y triptófano, definió a la pelagra como una enfermedad endémica, "poco frecuente en nuestro pueblo". ${ }^{64}$

Entre los sustentos de la descalificación del maíz y demás alimentos de las clases populares a lo largo de la primera mitad del siglo xx estaba la idea del "hambre crónica". Ésta surgió en la época del descubrimiento de América, cuando los españoles se percataron de que los indígenas carecían de ganado domesticado, no obstante, estos últimos contaban con una alimentación completa y balanceada. Ya el historiador Plutarco Naranjo ha explicado que la desnutrición entre los indígenas apareció a raíz de la conquista, como consecuencia del profundo trastorno social y tecnológico que ésta provocó. ${ }^{65}$ Sin embargo, como esa es una situación soslayada naturalmente por la historiografía colonial y, aplicando el principio de autoridad, por la historiografía de épocas posteriores, ${ }^{66}$ en la primera mitad del siglo xx la idea del hambre crónica fue fomentada no sólo por los médicos, sino por ingenieros, antropólogos y abogados. En 1901, el abogado Julio Guerrero pretendió explicar con base en esa idea el atraso social de las clases bajas así como sus supuestas actitudes criminales. ${ }^{67}$ Por todo ello, para los médicos era urgente conducirlas hacia la "alimentación racional, económica e higiénica" ${ }^{68}$

${ }^{63}$ Pilcher, op. cit., p. 147; Warman, op. cit., p. 162; 180.

${ }^{64}$ Se padecía en Yugoslavia, Egipto, la parte sureste de los Estados Unidos, África del Sur y en la península de Yucatán, "respetando el resto de nuestro territorio en forma relativa". Precisamente por eso, el objetivo del INN, al iniciar la segunda mitad del siglo xx, era investigar el por qué de la incidencia de ese mal en Yucatán. José Calvo de la Torre, "Problemas de la alimentación popular en México", en Medicina. Revista Mexicana, México, Escuela Nacional de Medicina, núm. 633, agosto 10 de 1951, 314 y 318.

${ }^{65}$ Plutarco Naranjo, "La comida andina antes del encuentro", en Janet Long, coord., Conquista y comida. Consecuencias del encuentro de dos mundos, México, unam, 1996, 37.

${ }^{66}$ Véase al respecto, Juan Pío Martínez, Conquista, hambre y salvación. Los indios de la Provincia de Ávalos, 1523-1750, Guadalajara, Universidad de Guadalajara, Campus Universitario de Los Lagos, 2003.

${ }^{67}$ Véase, Julio Guerrero, La génesis del crimen en México. Estudio de psiquiatría social, 2a ed., México, Porrúa, 1977 [1901], 148-149. También el ingeniero Norberto Aguirre se refirió al hambre crónica de las clases populares, véase "El maíz y su apóstol”, en Alemán y la comisión del maíz, México, Ruta, 1951, 41.

${ }^{68}$ Para la cita textual véase, Samuel Máynez Puente, "La alimentación del mexica- 
La urgencia de modificar los patrones alimenticios de las clases populares era generalizada en los países latinoamericanos. ${ }^{69}$ Para los médicos mexicanos eran los "hábitos viejos y costumbres ancestrales nocivas, las que urge corregir por todos los medios posibles" ${ }^{70}$ Una de esas prácticas que les urgía a los médicos corregir, era la costumbre de los campesinos y habitantes en general del medio rural, de que en lugar de consumir la leche, los pollos y los huevos que algunos de ellos producían, los vendieran "a precios irrisorios" para comprar "pulque en vez de leche y otros alimentos" ${ }^{71}$ Para los médicos mexicanos estaba claro sin lugar a dudas que sólo los alimentos de origen animal podían darle "potencia" a la raza. Por eso, desde una óptica racista, un informe de la Secretaría de Salubridad y Asistencia afirmaba la imposibilidad de ser un pueblo superior, "mientras 15 millones de mexicanos se alimentan a base de proteínas que son inadecuadas aun para bestias". De ahí que se considerara necesario, "modificar profundamente la alimentación de todos nuestros niños, introduciéndoles a toda costa cualquier alimento de origen animal (sangre de buey, pescado, leche, huevos, etc.)".$^{72} \mathrm{Al}$ parecer fue la leche el alimento "racional y científico" que hacia 1950 empezó a tener mayor presencia en la mesa de las clases populares, porque su

no", en La Prensa médica mexicana. Publicación mensual para la difusión de los conocimientos médicos, México, s.e., núms. 3 al 6, mayo-agosto de 1956, 89-90. Sobre los médicos, la idea del hambre crónica, y la necesidad de resolver el problema en el sentido señalado, véase: Miguel Bustos López, "México se alimenta mal e insuficientemente", en Medicina. Revista Mexicana, México, Escuela Nacional de Medicina, núm. 552, marzo 25 de 1948, 43; Ramos Espinosa, La alimentación en México, op. cit., p. 129; y del mismo autor, "Notas breves...", op. cit., p. 158.

${ }^{69}$ Véase, Jaime Pi-Suñer, "El problema económico de la alimentación”, op. cit., p. 406; y Escudero, op. cit., p. 81.

${ }^{70}$ Olascoaga y Ramos Espinosa, op. cit., p. 6; Ramos Espinosa, "El maíz, el frijol y el chile", op. cit., p. 17.

${ }^{71}$ José Quintín Olascoaga y Alfredo Ramos Espinosa, Bases para el cálculo de la alimentación correcta, México, Departamento de Salubridad Pública, Oficina General de Higiene de la Alimentación, Sección de Investigación de la Alimentación Popular, folleto número 13, 1940, 15; Salvador Bermúdez, Elementos de higiene, 3a. ed., México, s.e., s.a.p. 184; Ramos Espinosa, "La habitación y el vestido..." op. cit., p. 461; Calvo de la Torre, op. cit., p. 314.

${ }^{72}$ Archivo Secretaría de Salubridad y Asistencia, fondo ssa, scción SubSyA, caja 17, exp. 11 . 
aceptación iba resultando "más fácil que la de otros alimentos de origen animal", especialmente entre los niños y sus madres, que fueron los principales destinatarios de ese consumo. ${ }^{73}$

\section{Conclusiones}

La creación de una nueva necesidad alimenticia para la mayor parte de mexicanos, como la del consumo de leche de vaca, prueba que los nutriólogos contribuyeron al control social de las masas divulgando los principios de la ciencia de la nutrición. Persuadidos de que eran el medio más adecuado para civilizar a la población de más bajos recursos, soslayaron que la aplicación de esos principios entraba en franca contradicción con las prácticas alimenticias de ésta, ya que a lo largo de la primera mitad del siglo xx más del cincuenta por ciento de los habitantes del país seguía apegado al modelo de alimentación indígena. Esa circunstancia generó la premura de las clases gobernantes de concluir el proceso de civilización iniciado desde la época de la conquista, lo que pone de manifiesto que seguía vigente la confrontación entre ese modelo de alimentación, cuya dieta básica era maíz, chile, frijoles y pulque, y el modelo de alimentación occidental, basado en carne, pan y, ya entrado el siglo xx, leche de vaca. Ciertamente son modelos ideales, pero se puede hacer referencia a ellos porque fueron construidos por las mismas fuentes consultadas para realizar este artículo.

Bajo argumentos de carácter científico se trató de convencer de la superioridad nutricional del modelo de alimentación occidental sobre el modelo de alimentación indígena. Sin embargo, más allá de esa discusión netamente nutriológica, es preciso destacar dos aspectos de esa confrontación. El primero tiene que ver con las diferencias culturales entre lo indígena y lo europeo. Para los grupos sociales identificados consanguíneamente o por tradición cultural con lo indígena, sus prácticas alimenticias eran suficientes y adecuadas -siempre y cuando las condiciones socioeconómicas y políticas

${ }^{73}$ Jesús Díaz Barriga, "Nutrición en la República Mexicana”, en Medicina. Revista Mexicana, México, Escuela Nacional de Medicina, núm. 619, enero 10 de 1951, 1 y 4. 
de la primera mitad del siglo xx se los permitieran-, igual que lo eran las suyas para los europeos y los criollos, incluyendo a los mestizos occidentalizados. El segundo aspecto involucra la historicidad de los modelos confrontados, particularmente la del modelo de alimentación occidental. Para los nutriólogos europeos y mexicanos la defensa de la corriente carnívora como factor de civilización y progreso resultaba inherente a su peculiar cosmovisión, pues la hegemonía cultural subyacente al desarrollo de la ciencia de la nutrición validaba esa perspectiva. Por eso se consideraba como natural y necesario lo que de entrada constituía algo socialmente determinado. Así se velaron en su momento las relaciones ideológicas y de poder que mantuvieron dividida a la sociedad entre carnívoros y vegetarianos, hasta que supuestamente el capitalismo terminó con los privilegios que generaban esa división.

Si bajo el nuevo sistema socioeconómico las clases bajas del sector urbano, tanto europeas como mexicanas, pudieron acceder a un mayor consumo de carne, esto no eliminó la cualidad de símbolo de prestigio y estatus social alto atribuido a este alimento desde la época medieval. Antes al contrario, mantuvo vigente a la ideología alimentaria surgida también desde aquellos tiempos. Por esa razón resultó ambigua la postura de los nutriólogos mexicanos y del Estado en cuanto a la corriente carnívora, pues para exaltarla y de paso desprestigiar al maíz sugerían añadirle carne a la dieta a base de ese cereal, pero no promovieron campańas que les permitieran a las clases de bajos recursos acceder cotidianamente al consumo de carne. No al menos como sí lo hicieron con la leche de vaca, cuyo consumo empezó a masificarse al terminar la primera mitad del siglo xx, lo que permite hablar de la creación de una nueva necesidad dietética, aunque divulgada como si de una necesidad natural se tratara. Quizá para las poblaciones europeas era natural y necesario el consumo de leche de vaca, pero no era lo mismo en el caso de la mayor parte de la población mexicana. El proceso de introducción de ese producto en el modelo de alimentación indígena no tuvo en cuenta las diferencias culturales ni la desadaptación fisiológica de la mayor parte de los mexicanos para consumir ese alimento. No obstante, las perspectivas nutriológicas occidentalizadas tienden a minimizar la 
relevancia de esos aspectos. Por lo tanto, tampoco se ha considerado lo suficiente el papel del monopolio de la ganadería bovina, y mucho menos los intereses económicos implicados en la creación de esa nueva necesidad en México -y en otras partes del mundo no occidental- encubiertos bajo el pretexto de resolver lo que desde una perspectiva dominante se consideran deficiencias nutricionales.

Así se ha ejercido el control social, pues en lugar de respetarse y fomentarse las respectivas soberanías alimentarias, se ha tratado de universalizar el modelo de alimentación occidental y las consecuentes formas de desarrollo económico, en lo que ha desempeñado un papel fundamental la ciencia de la nutrición.

\section{FuENTES CONSULTADAS}

Archivo Secretaría de Salubridad y Asistencia, DF, fondo SSA, sección SubSyA, caja 17, exp. 11.

Aguilar Rodríguez, Sandra, "Cooking modernity: nutrition policies, class, and gender in 1940s and 1950s Mexico city", en The Americas, 64:2 octubre 2007.

"Alimentando a la nación: género y nutrición en México (1940-1960)”, en Revista de Estudios Sociales, núm. 29, Universidad de los Andes, abril 2008. Texto disponible en internet: http://res.uniandes.edu.co/view.php/425/1.php

Aguilera Ríos, Sara y Juan José Saldaña, "Estado, comunidad médica e institucionalización de los estudios sobre nutrición en México, (1937-1957)", en Juan José Saldaña, coord., La Casa de Salomón en México. Estudios sobre la institucionalización de la docencia y la investigación científicas, México, Facultad de Filosofía y Letras, UNAM, 2005.

Aguirre Beltrán, Gonzalo, Obra antropológica v. Programas de salud en la situación intercultural, México, Universidad Veracruzana, Instituto Nacional Indigenista, Gobierno del Estado de Veracruz, Fondo de Cultura Económica, 1994 [Primera edición, III, 1955] Aguirre, Norberto, "El maíz y su apóstol", en Alemán y la comisión del maiz, México, Ruta, 1951.

BengoA, José María, "Nutrición en América Latina: algunos esla- 
bones de su historia”, en Héctor Bourges R., José M. Bengoa y Alejandro M. O'Donnell, coords., Historias de la nutrición en América Latina, sLAN Sociedad Latinoamericana de Nutrición, Publicación sLan número 1, Fundación Cavendes, INCMNSZ Instituto Nacional de Ciencias Médicas de la Nutrición Salvador Zubirán, CESNI Centro de Estudios Sobre Nutrición Infantil. Texto disponible en Internet: http://www.slan.org.mx/docs/ HistNut_SLAN.pdf

Bermúdez, Salvador, Elementos de higiene, 3a. ed., México, s.e., s.a. Bernabeu-Mestre, Josep et al., "La alimentación como problema sanitario: nutrición y salud pública en la Espańa de la primera mitad del siglo xx", en VIII Congreso de la Asociación de Demografia Histórica. 30 de mayo, 1 y 2 de junio 2007, Maó (Menorca). Texto disponible en Internet: http://www.redmei.org/index. php?option=com_docman\&task=cat_view\&gid=69\&dir=DES C\&order=date\&Itemid $=45 \&$ limit $=5 \&$ limitstart $=10$

Bouant, Emilio, Nuevo diccionario de quimica aplicada a las ciencias, a las artes, a la agricultura, a la industria y a la farmacia, tomo I, Traducido, anotado con datos referentes a España y aumentado con gran número de artículos bajo la dirección de los señores Ramón de Manjarrés y Bofarull y Federico Trémols y Borrell; con la colaboración de Juan Antonio Vidal de Solano, Casimiro Brugués y Escuder y Ramón de Manjarrés y PérezJunguitu, Barcelona, Espasa y Compañía, editores, s.a.

Brillat-SaVArin, Jean Anthelme, Fisiología del gusto, Barcelona, Espańa, Optima, 2001.

BRUGUÉs, Casimiro, Quimica popular, 3a. ed., con un prólogo de José Casares, Barcelona, Gustavo Gili, editor, 1918.

Bustos López, Miguel, "México se alimenta mal e insuficientemente", en Medicina. Revista Mexicana, México, Escuela Nacional de Medicina, núm. 552, marzo 25 de 1948.

Calvo de la Torre, José, "Problemas de la alimentación popular en México", en Medicina. Revista Mexicana, México, Escuela Nacional de Medicina, núm. 633, agosto 10 de 1951.

Cantala, Julio, El insipido. (Panorama del hombre civilizado), Nueva York, Press Service, Inc., 1941. 
Cendrero Curiel, Orestes, Elementos de higiene, 6a. ed., Santander, s.e., 1926.

Corcuera de Mancera, Sonia, Entre gula y templanza. Un aspecto de la historia mexicana, 2a. ed., México, Fondo de Cultura Económica, 1990.

Cravioto, René O., "Contribución al conocimiento del valor nutritivo de los alimentos mexicanos: el maíz", en Medicina. Revista Mexicana, México, Escuela Nacional de Medicina, núm. 584, julio 25 de 1949.

Cravioto, Rene O., P. G. Massieu, Jesús Guzman G., "El problema de las proteínas en la dieta mexicana", en Boletín de la Oficina Sanitaria Panamericana, febrero 1955. Texto disponible en Internet: http://hist.library.paho.org/Spanish/BOL/v38n2p148. pdf

Chapin, Steven, "Historia y debate del vegetarianismo: sus raíces religiosas, médicas y filosóficas”. Texto disponible en Internet: http://www.librosperuanos.com/traducciones/esquina32.html

Devés, Eduardo y Ricardo Melgar, "El pensamiento del Asia en América Latina. Hacia una cartografía”, en Revista de Hispanismo Filosófico, núm. 10, 2005. Texto disponible en Internet: http://descargas.cervantesvirtual.com/servlet/SirveObras/0159 3630980142818560035/022815.pdf?incr $=1$

DíAz BARriga, Jesús, "Nutrición en la República Mexicana”, en Medicina. Revista Mexicana, México, Escuela Nacional de Medicina, núm. 619, enero 10 de 1951.

Elias, Norbert, El proceso de la civilización. Investigaciones sociogenéticas y psicogenéticas, 2a ed., trad. Ramón García Cotarelo, México, Fondo de Cultura Económica, 1994.

Escudero, Pedro, La alimentación. Colaboraciones publicadas en La Prensa, Buenos Aires, Hachette, 1934.

Estadisticas históricas de México, tomo I, $4^{\mathrm{a}}$ ed. Aguascalientes, INEGI, 2000.

FEDER, Ernest, "La irracional competencia entre el hombre y el animal por los recursos agrícolas de los países subdesarrollados", en El trimestre económico, México, Fondo de Cultura Económica, núm. 185, enero-marzo de 1980. 
Fernández y Fernández, Ramón, "Las posibilidades agrícolas de México", en Universidad mensual de cultura popular, México, Universidad Nacional, núm. 10, noviembre de 1936.

Gamio, Manuel, Antología, Estudio preliminar, selección y notas por Juan Comas, 2a ed., México, UnAM, 1985.

García Garibay, Mariano y Agustín López Munguía Canales, "Enzimas inmovilizadas y su aplicación en la industria alimentaria”, en Ciencia y Desarrollo, México, Conacyt, núm. 58, año x, septiembre-octubre de 1984.

García, Rubén, "Hambre y maíz. Síntesis del artículo del Gral. [...] publicado en El Universal", en Guión, México, s.e., núm. 5, 1 de marzo de 1939.

Gerardin, León, El hombre. Elementos de fisiologia, de higiene, de prehistoria y de etnografía, versión castellana, Paris, México, Librería de CH. Bouret, 1903.

Giral Pereira, José, Ración alimenticia desde los puntos de vista higiénico y social, Madrid, Librería Internacional, Adrián Romo, editor, 1914.

Guerrero, Julio, La génesis del crimen en México. Estudio de psiquiatría social, 2a ed. México, Porrúa, 1977 [1901].

HARris, Marvin, Bueno para comer. Enigmas de alimentación y cultura, Colección Los Noventa, 75, México, Alianza, Conaculta, 1991.

Heraldo Naturista, El, Revista ilustrada de México, México, s.e., 1921-1922; 1923-1924.

Historia de la salubridad y de la asistencia en México, tomos II y III, México, Secretaría de Salubridad y Asistencia, 1960.

Hogar, El, México, Compañía Editora Mexicana, núm. 1066, 13 de marzo de 1941.

Janowitz, Morris, “Teoría social y control social”, Traducción de Juan Pegoraro, revisión de Máximo Sozzo, en American Journal of Sociology, vol. 81, 1, Universidad de Chicago, 1975. Publicado en Delito y Sociedad. Revista de Ciencias Sociales, núm. 6/7, 1995. Texto disponible en internet: http://www.catedras.fsoc. uba.ar/pegoraro/Materiales/Janowitz_Teoria_Social_Control_ Social.PDF 
Maestro, El, Revista de Cultura Nacional, México, Universidad Nacional, 1921, 1922.

Máynez Puente, Samuel, "La alimentación del mexicano", en $\mathrm{La}$ Prensa médica mexicana. Publicación mensual para la difusión de los conocimientos médicos, México, s.e., núms. 3 al 6, mayo-agosto de 1956.

Millán, Amado, "Acerca del status animal", en Ricardo Avila, Dominique Fourier y Ma. Teresa Ruiz, comps., Estudios del Hombre 7, Guadalajara, Universidad de Guadalajara, Departamento de Estudios del Hombre, 1998.

Miranda, Francisco de P., La alimentación en México, México, Instituto Nacional de Nutriología, 1947.

Montanari, Massimo, El hambre y la abundancia. Historia y cultura de la alimentación en Europa, trad. Juan Vivanco, Barcelona, España, Crítica, Grijalbo, 1993.

Naranjo, Plutarco, "La comida andina antes del encuentro", en Janet Long, coord., Conquista y comida. Consecuencias del encuentro de dos mundos, México, UnAM, 1996.

Newton, Evans, Percy T. Magan y George Thomason, El nuevo médico de la familia. Tratado sobre la prevención y curación de las enfermedades; no destinado a reemplazar al médico de la familia, sino a ayudar al lector a cooperar más inteligentemente con él, (Traducida al castellano por Marcelo I. Fayard y revisada por el Dr. Carlos E. Westphal), Mountain View, Pacific Press Publishing Association, 1925.

Olascoaga, José Quintín, "Bases para los estudios de bromatología y nutrición en la República Mexicana”, en Medicina. Revista Mexicana, México, Escuela Nacional de Medicina, núm. 348, septiembre 25 de 1939.

, "Datos para la historia de la nutriología en México", en Medicina. Revista Mexicana, México, Escuela Nacional de Medicina, núm. 573, febrero 10 de 1949.

Olascoaga, José Quintín y Alfredo Ramos Espinosa, Bases para el cálculo de la alimentación correcta, México, Departamento de Salubridad Pública, Oficina General de Higiene de la Alimentación, Sección de Investigación de la Alimentación Popular, folleto número 13, 1940. 
Oliver Olmo, Pedro, "El concepto de control social en la historia social: estructuración del orden y respuestas al desorden", en Historia Social, núm. 51, 2005, 73-91. Texto disponible en Internet: http://www.uclm.es/profesorado/poliver/pdf/articulos/ HistoriaSocial.pdf

Pascault, L., La salud por la alimentación racional, Traducción del francés: José Roda Rodríguez, 3a ed. Barcelona, Librería de D. Ribó Pelayo, 1915.

Pi-Suñer, Jaime, "El problema económico de la alimentación", en Atenea. Revista Mensual de Ciencias, Letras y Artes, Santiago de Chile, Universidad de Concepción, núm. 79, septiembre de 1931. , Las bases fisiológicas de la alimentación, México, Fondo de Cultura Económica, La Casa de España en México, 1940.

Pilcher, Jeffrey, ; Vivan los tamales! La comida y la construcción de la identidad mexicana, trad. Victoria Schussheim, México, Ediciones de la Reina Roja, Conaculta, Ciesas, 2001.

Pío Martínez, Juan, Conquista, hambre y salvación. Los indios de la Provincia de Ávalos, 1523-1750, Guadalajara, Universidad de Guadalajara, Campus Universitario de Los Lagos, 2003.

Plasencia, Pedro, A la mesa con don Quijote y Sancho, España, Punto de lectura, 2005.

Quintana, Epaminondas, "El ingente problema del maíz en su aspecto agrícola y nutritivo”, en América indígena, s.p.i., núm. 2, abril 1944.

Ramos Espinosa, Alfredo, "La habitación y el vestido en el campo.La alimentación popular y los procesos mentales", en Medicina. Revista Mexicana, México, Escuela Nacional de Medicina, número 252, septiembre 25 de 1935.

, "Notas breves sobre nuestras posibilidades de alimentación”, en Medicina. Revista Mexicana, México, Escuela Nacional de Medicina, núm. 265, abril 10 de 1936.

"Valor energético medio de la alimentación en México", en Medicina. Revista Mexicana, México, Escuela Nacional de Medicina, núm. 292, mayo 25 de 1937.

, La alimentación en México, México, s.e., 1939.

,"El maíz, el frijol y el chile", en CAMEP. La prensa médica 
mexicana, México, Centro de Asistencia Médica para Enfermos Pobres, núm. 2, sábado 15 de febrero de 1941.

, "En torno al estudio geográfico de la alimentación en México", en Medicina. Revista Mexicana, México, Escuela Nacional de Medicina, núm. 440, julio 25 de 1943.

, "El folklore y la alimentación", en Anuario de la sociedad folklórica de México. 1941, vol. II, México, Círculo Panamericano de Folklore, 1943.

"Mi contribución al estudio de la alimentación mexicana", en Medicina. Revista Mexicana, México, Escuela Nacional de Medicina, tomo xxviII, núm. 560, julio 25 de 1948.

, "Industrialización del maíz", en Medicina. Revista mexicana, México, Escuela Nacional de Medicina, núm. 586, agosto 25 de 1949.

Reuleaux, Francisco, Los grandes inventos en todas las esferas de la actividad humana y sus principales aplicaciones cientificas, artisticas, industriales, comerciales y domésticas, tomo v, Primera traducción española, hecha de la octava edición alemana por Federico Gillman, Madrid, Gras y Compañía, editores, 1888.

Revista de Investigación Clinica. Órgano del Hospital de Enfermedades de la Nutrición, México, Hospital de Enfermedades de la Nutrición, núm. 1, octubre de 1948.

Ritchie, Carson I.A., Comida y civilización. De cómo los gustos alimenticios han modificado la historia, trad. José Carlos Gómez Borrero, Madrid, Alianza Editorial, 1988.

Romero, José Luis, Estudio de la mentalidad burguesa, México, Alianza, 1989.

Rosen, George, $A$ history of public health, Nueva York, MD Publications, Inc., 1958.

Schor, Isaias y Saul Senderey, "Nutrición siglo xx. Bosquejo histórico de la nutrición en la República argentina”, en Héctor Bourges R., José M. Bengoa, Alejandro M. O’Donnell, coords., Historias de la nutrición en América Latina, sLAN Sociedad Latinoamericana de Nutrición, Publicación sLAN número 1, Fundación Cavendes, InCMnsz Instituto Nacional de Ciencias Médicas de la Nutrición Salvador Zubirán, CESNI Centro de Es- 
tudios Sobre Nutrición Infantil, Texto disponible en Internet: http://www.slan.org.mx/docs/HistNut_SLAN.pdf

Sherman, Henry C., La ciencia de la nutrición, versión española de Germán Somolinos, México, Fondo de Cultura Económica, 1945.

Treviño, Norberto, "Alimentación en los trabajadores", en CAMEP. La prensa médica mexicana, México, Centro de Asistencia Médica para Enfermos Pobres, núm. 12, 1 de noviembre de 1936. Turner, Bryan S., "The discourse of diet", en Mike Featherstone, Mike Hepworth y Bryan S. Turner, eds., The body social process and cultural theory, Londres, Sage Publications, 1991.

Warman, Arturo, La historia de un bastardo: maiz y capitalismo, México, Fondo de Cultura Económica, UnAM, 1988.

Wirz, Franz G.M., "Política sanitaria de la alimentación", en Wilhelm Stepp, dir., Fisiología, patología, terapéutica y politica sanitaria de la alimentación. Las vitaminas, versión directa del alemán por el Dr. Julio G. Sánchez Lucas, Barcelona, Labor, 1942.

FeCha de RECEPCIÓN DEL ARTículo: 8 de marzo de 2010 FECHA DE RECEPCiÓN DE LA VERSIÓN FINAL: 19 de julio de 2011 